\title{
SCLOUDPY: Sistema Informático Web de Multi-Tenencia para el Procesamiento en la Nube de Pedidos de PYMES
}

\author{
Martha H. Carrillo(1), Consuelo Franky(2) ,Pablo S. Páez ${ }^{(3)}$ y Andrés F. Pedraza ${ }^{(4)}$ \\ (1) Departamento de Ingeniería Industrial, Pontificia Universidad Javeriana, Carrera 7 No 40-62, Bogotá- \\ Colombia (e-mail: mcarrill@javeriana.edu.co) \\ (2)(3)(4)Departamento de Ingeniería de Sistemas, Pontificia Universidad Javeriana, Carrera 7 No 40-62, \\ Bogotá-Colombia (e-mail:Ifranky@javeriana.edu.co; pasepama@hotmail.com; \\ pedraza.andres@javeriana.edu.co)
}

Recibido Jul. 10, 2015; Aceptado Sep. 10, 2015; Versión final Oct. 29, 2015, Publicado Feb. 2016

\begin{abstract}
Resumen
El presente artículo tiene como objetivo describir mejoras introducidas a SCLOUDPY, un sistema web de operación de flujos de pedidos en las cadenas de suministro. La idea es lograr su ampliación a un sistema de tenencia múltiple (multi-tenencia) lo cual permite operar el sistema en modo Computación en la Nube (Cloud Computing). Esto permite atender a múltiples pequeñas y medianas empresas (PYMES) a la vez, asegurando al mismo tiempo la independencia de datos de estas PYMES. Para lograr estas mejoras se aplicaron varias metodologías como DAR - Análisis y toma de decisiones (Decision Analysis and Resolution) para seleccionar los requerimientos más relevantes, y la metodología ágil XP para la construcción del software. Se logró un sistema de tenencia múltiple que opera en la nube, que satisface las necesidades de múltiples PYMES al tiempo y que además no representa mayores costos por haber sido construido con herramientas de software libre. Esta combinación de modelo de operación y arquitectura de software asegura un sistema de procesamiento de pedidos eficiente y flexible.
\end{abstract}

Palabras clave: cadena de suministro; gestión de pedidos; computación en la nube; multi-tenencia; modelado; PYMES

\section{SCLOUDPY: Multi-Tenant and Cloud Web Information System for Orders Processing in SMEs}

\begin{abstract}
This article aims to describe the improvements made to SCLOUDPY, a web system of order flows management in supply chains. The idea is to achieve its expansion to a multitenant system which allows the system to operate in Cloud Computing mode. This allows serving several small and medium size enterprises (SMEs) ensuring the independence of data from these SMEs. To achieve these improvements various methodologies were applied as a DAR assessment (Decision Analysis and Resolution) to select the most relevant requirements, and XP agile methodology for software construction. Finally, a multitenant system that operates in the Cloud that meets the needs of several SMEs was obtained. At the same time the system does not represent additional costs because the system was built with free software. This combination of operating model and software architecture ensures an efficient and flexible system for processing orders.
\end{abstract}

Keywords: supply chain; order management; cloud computing; multitenant; modeling; SME 


\section{INTRODUCCIÓN}

Los actuales sistemas de apoyo informático para el procesamiento de pedidos requieren bastantes recursos para su implementación y mantenimiento así como la exigencia de una excelente infraestructura de IT, aspectos generalmente fuera del alcance de las Pymes (Zare, 2010) que ven afectados sus procesos por la carencia de estas herramientas (You, 2011) (Antoniadis, 2014). Adicionalmente como existen muy pocos estudios para este tipo de empresas, nuevos aportes en este sentido tienen relevancia (Chike y Xiong, 2009) (Ruivo y otros, 2013) (Cabarcas et al., 2015).

Mediante proyectos patrocinados se desarrolló durante los años 2013 y 2014, SCLOUDPY: Sistema en la Nube para la gestión del flujo de pedidos de pymes manufactureras (Carrillo y Franky, 2014). Se partió del diseño de un nuevo modelo referencial en el dominio de flujo de pedidos que presenta alternativas para superar los problemas de los modelos tradicionales secuenciales (Garcia y You, 2015) (Aguilar-Saven, 2004) (Amer et al., 2009). Se utilizaron herramientas de software libre y el concepto lógico de red para conectar a las entidades involucradas a través de una infraestructura de Computación en Nube (Cloud Computing) por las ventajas que esta opción presenta (Carrillo y Franky, 2014). Adicionalmente se agregó el soporte de "multi-tenencia", muy adecuado para los sistemas que operan en la Nube, el cual es un tipo de arquitectura de software en el cual una sola aplicación da servicio a múltiples empresas clientes ofreciendo una visión de datos particionados en donde cada empresa solo ve sus datos y no los de otra, así todos los datos convivan en una misma base de datos. Posteriormente se construyó el correspondiente soporte informático, de modo que ambos: el modelo y su soporte, conforman el modelo final. Las ventajas que ofrece SCLOUDPY son: actividades no secuenciales, independencia de actividades, operación en la nube, visibilidad, disminución del riesgo de fallo, costos bajos para pymes por apoyarse en herramientas de software libre, y flujos de información colaborativos. (Carrillo y Franky, 2014).

Dentro del proceso de validación final que se realizó, se detectó que el sistema necesitaba contar con algunas funcionalidades adicionales para que pudiera adaptarse a la realidad de la operación de las pymes (Carrillo y Franky, 2014). Se definió entonces realizar un proceso de revisión de autores que presentaran alternativas al respecto así como una verificación de publicaciones de sistemas actuales similares que junto con la validación empresarial permitiera definir las posibles funcionalidades requeridas mediante una metodología apropiada y posteriormente proceder a su desarrollo. El presente artículo tiene como objetivo describir estas mejoras introducidas al sistema SCLOUDPY. En las siguientes secciones se presentará una comparación del sistema SCLOUDPY con otros trabajos resaltando su contribución, un análisis de funcionalidades adicionales para SCLOYPY y la selección de esas funcionalidades. Posteriormente se describirá la arquitectura del sistema SCLOUDPY y la validación realizada para este sistema. Finalmente se presentarán las conclusiones de este trabajo.

SCLOUDPY como sistema que opera bajo el modelo de operación en la Nube goza de las ventajas que trae este tipo de tecnología, permitiendo un uso óptimo de recursos mediante la reconfiguración dinámica del sistema de acuerdo a las necesidades de la empresa o empresas que utilizan el sistema (Cegielski et al., 2012). Para los sistemas de Procesamiento de pedidos, que son complejos por naturaleza, el modelo de operación en la Nube ofrece flexibilidad como principal ventaja pues puede escalar en poder computacional sin incurrir en altos costos y permite lograr un sistema más efectivo y eficiente; adicionalmente el modelo de operación en la Nube facilita la comunicación, coordinación y colaboración entre las múltiples empresas que participan en el Procesamiento de pedidos (Cegielski et al., 2012).

Existe un interés creciente por implantar sistemas de Procesamiento de pedidos con soporte en Internet como lo muestran los trabajos: (Christopher et al., 2004), (Boyer y Hult, 2005) y (Lindner et al., 2010). Por otro lado hay estudios de cómo el modelo de computación en la Nube puede traer grandes beneficios a los sistemas de Procesamiento de pedidos, como lo describen (Cegielski et al., 2012) y (Kshetri, 2010). La contribución principal del proyecto descrito en este artículo acerca del sistema SCLOUDPY es unir las 2 tendencias mencionadas, es decir montar el sistema sobre el soporte de red que da Internet y adicionalmente poner a operar el sistema en modo Computación en la Nube. En cuanto a la arquitectura de software de Tenencia múltiple, son pocos los sistemas de Procesamiento de pedidos que se implantan siguiendo esta arquitectura como lo hace el sistema SCLOUDPY (ver por ejemplo (Lin et al., 2011), lo cual constituye un aporte importante del proyecto descrito en este artículo.

\section{ANALISIS DE FUNCIONALIDADES ADICIONALES DEL SISTEMA SCLOUDPY}

A partir de la validación de la primera versión de SCLOUDPY (Carrillo y Franky, 2014) se determinaron algunos de los posibles requerimientos faltantes que podrían ser del interés de las Pymes. Adicionalmente, en los últimos años autores como Su y Yang (2010), Hsu ( 2013), Ruivo et al., ( 2015), Aslan et al., (2012) han hecho aportes en cuanto a las posibles funcionalidades de un sistema ERP entendido éste como una 
aplicación de software que automatiza e integra tanto los procesos del manejo de un negocio así como la producción y distribución y que generalmente incluye aplicaciones para el manejo de pedidos.

Con el fin de complementar esta información se realizó una investigación no estructurada en cuanto a los sistemas de software existentes en el mercado para el procesamiento de pedidos (Jacobs y Weston, 2007) (Elragal y Haddarab, 2012) y de allí se extrajeron otros posibles requerimientos a considerar basados en publicaciones de medios especializados. En la tabla 1 aparecen las fuentes. La tabla 2 resume los requerimientos que se consideraron e incluye columnas sobre el origen del requerimiento y tipo, con las siguientes convenciones:

Origen del requerimiento: $(\mathrm{T})$ proviene de la validación desarrollada para el sistema SCLOUDPY (Carrillo y Franky, 2014) en una investigación anterior; (B) proviene de artículos sobre el tema del estudio o de publicaciones generales sobre sistemas de software existentes en el mercado para el procesamiento de pedidos.

Tipo de requerimiento (Bruegge, 2002): (F) Funcional: define qué hace el sistema (describen entradas y salidas), es decir, se refiere a las funciones que debe realizar el sistema; (NF) No funcional: definen cómo el sistema debe realizar su trabajo (en cuanto a eficiencia, hardware, software, interfaces con otros sistemas, seguridad, utilización, etc.).

Tabla 1. Fuentes de análisis de sistemas de software en el mercado para el procesamiento de pedidos

\begin{tabular}{|c|c|}
\hline $\begin{array}{l}\text { Relacionados con sistemas de software en } \\
\text { general }\end{array}$ & $\begin{array}{l}\text { Relacionados con sistemas de software de } \\
\text { diferentes países }\end{array}$ \\
\hline $\begin{array}{l}\text { Logility Voyage Solutions(Logility,2015), } \\
\text { JDA Demand(JDA,2012), } \\
\text { JDA Fulfillment(JDA,2012), } \\
\text { Advanced Planning(Logismarket,2013), } \\
\text { SAP SCM(SAP,2015), } \\
\text { Openbravo (Openbravo,2012), }\end{array}$ & $\begin{array}{l}\text { Rusia (IPO Board,2013), } \\
\text { España (EPICOR,2015), } \\
\text { Japón (Yoshihara,2015), } \\
\text { Brasil (OpenERP,2015), } \\
\text { USA (Asociación,2015) }\end{array}$ \\
\hline $\begin{array}{l}\text { Neogia (Neogia,2012), } \\
\text { OpenERP (openERP, 2015), }\end{array}$ & $\begin{array}{l}\text { Relacionados con sistemas de software en } \\
\text { Latinoamérica }\end{array}$ \\
\hline $\begin{array}{l}\text { ERP5 (ERP5,2012), } \\
\text { Adempiere (adempiere,2015), } \\
\text { OMS Order Dynamics (Dynamics,2015), } \\
\text { Ability OMS (ability,2013) }\end{array}$ & $\begin{array}{l}\text { Productivos LTDA (productivos,2015), } \\
\text { SIIGO (Siigo,2015), OasisIT (OasisCom,2013) }\end{array}$ \\
\hline
\end{tabular}

Tabla 2: Resumen de Requerimientos del sistema SCLOUDPY

\begin{tabular}{|l|l|l|c|c|}
\hline Requerimiento & \multicolumn{1}{|c|}{ Descripción } & Tipo & Origen & Selección \\
\hline REQF-001 & SCLOUDPY debe permitir la gestión de inventarios para PYMEs. & $F$ & $T$ & \\
\hline REQF-002 & $\begin{array}{l}\text { SCLOUDPY debe permitir a una PYME hacer pedidos de productos } \\
\text { por lotes a un proveedor. }\end{array}$ & $F$ & $T$ & $\mathrm{X}$ \\
\hline REQF-003 & $\begin{array}{l}\text { SCLOUDPY debe permitir que una PYME pueda seleccionar un } \\
\text { proveedor para el pedido que desee realizar. }\end{array}$ & $F$ & $T$ & $\mathrm{X}$ \\
\hline REQF-004 & $\begin{array}{l}\text { SCLOUDPY debe implementar un módulo de contabilidad básico que } \\
\text { permita persistir los ingresos y los gastos de una PYME. }\end{array}$ & $F$ & $B$ & \\
\hline REQF-005 & $\begin{array}{l}\text { SCLOUDPY debe permitir la generación de reportes contables para } \\
\text { analizar el estado de ingresos y egresos de una PYME. }\end{array}$ & $F$ & $B$ & \\
\hline REQF-006 & $\begin{array}{l}\text { SCLOUDPY debe implementar un módulo de estadística que permita } \\
\text { a un proveedor determinar si un pedido hecho por una PYME puede } \\
\text { realizarse o no, según pedidos históricos. }\end{array}$ & $F$ & $B$ & \\
\hline REQF-007 & $\begin{array}{l}\text { SCLOUDPY debe permitir a una PYME asociarse con múltiples } \\
\text { proveedores. }\end{array}$ & $F$ & $B$ & $X$ \\
\hline REQF-008 & SCLOUDPY debe permitir a una PYME eliminar un cliente. & $F$ & $B$ & $X$ \\
\hline REQF-009 & SCLOUDPY debe permitir la creación de un distribuidor. & $F$ & $B$ & $X$ \\
\hline
\end{tabular}


Tabla 2: (continuación)

\begin{tabular}{|c|c|c|c|c|}
\hline REQF-010 & SCLOUDPY debe permitir la autenticación de un distribuidor. & $F$ & $B$ & $X$ \\
\hline REQF-011 & SCLOUDPY debe permitir la creación de un productor. & $F$ & $B$ & $\mathrm{X}$ \\
\hline REQF-012 & SCLOUDPY debe permitir la autenticación de un productor. & $F$ & $B$ & $\mathrm{X}$ \\
\hline REQF-013 & $\begin{array}{l}\text { SCLOUDPY debe permitir a una PYME la adición de bodegas } \\
\text { contenedoras de productos. }\end{array}$ & $F$ & $B$ & \\
\hline REQF-014 & $\begin{array}{l}\text { SCLOUDPY debe permitir a una PYME la eliminación de bodegas } \\
\text { contenedoras de productos. }\end{array}$ & $F$ & $B$ & \\
\hline REQF-015 & $\begin{array}{l}\text { SCLOUDPY debe permitir a una PYME la edición de productos que } \\
\text { contiene una bodega. }\end{array}$ & $F$ & $B$ & \\
\hline REQF-016 & $\begin{array}{l}\text { SCLOUDPY debe permitir la persistencia de empleados que trabajan } \\
\text { en una PYME. }\end{array}$ & $F$ & $B$ & \\
\hline REQF-017 & $\begin{array}{l}\text { SCLOUDPY debe permitir la eliminación de empleados de una } \\
\text { PYME. }\end{array}$ & $F$ & $B$ & \\
\hline REQF-018 & $\begin{array}{l}\text { SCLOUDPY debe contar con un histórico de ventas para gestionar la } \\
\text { interacción con los clientes de la PYME. }\end{array}$ & $F$ & $B$ & \\
\hline REQF-019 & $\begin{array}{l}\text { SCLOUDPY debe permitir dar una descripción a los productos } \\
\text { ("Datos maestros del producto"). }\end{array}$ & $F$ & $B$ & $x$ \\
\hline REQF-020 & $\begin{array}{l}\text { SCLOUDPY debe mostrar información histórica de los productos } \\
\text { (cuando fue vendido, cantidad, si estuvo en promoción, agotado). }\end{array}$ & $F$ & $B$ & \\
\hline REQF-021 & $\begin{array}{l}\text { SCLOUDPY debe permitir que un cliente se asocie con múltiples } \\
\text { PYMEs. }\end{array}$ & $F$ & $B$ & $x$ \\
\hline REQF-022 & $\begin{array}{l}\text { SCLOUDPY debe permitir poner propiedades a los productos, por } \\
\text { ejemplo si un producto se encuentra en promoción. }\end{array}$ & $F$ & $B$ & \\
\hline REQF-023 & $\begin{array}{l}\text { SCLOUDPY debe implementar un calendario parametrizable por la } \\
\text { PYME, indicando qué días son laborables. }\end{array}$ & $F$ & $B$ & $x$ \\
\hline REQF-024 & $\begin{array}{l}\text { SCLOUDPY debe permitir parametrizar a una PYME un tiempo de } \\
\text { espera por pedido hecho a un proveedor. }\end{array}$ & $F$ & $B$ & \\
\hline REQF-025 & $\begin{array}{l}\text { SCLOUDPY debe permitir conjuntar las órdenes de un mismo } \\
\text { producto solicitadas por diferentes PYMES. }\end{array}$ & $F$ & $B$ & $x$ \\
\hline REQF-026 & $\begin{array}{l}\text { SCLOUDPY debe implementar un sistema BRMS (Business Rule } \\
\text { Management System) para controlar decisiones complejas de } \\
\text { negocio. }\end{array}$ & $F$ & $B$ & \\
\hline REQF-027 & $\begin{array}{l}\text { SCLOUDPY debe implementar una interfaz web que informe a una } \\
\text { PYME del sistema de manera rápida el estado de su inventario. }\end{array}$ & $F$ & $T$ & \\
\hline REQNF-028 & $\begin{array}{l}\text { SCLOUDPY debe implementar algoritmos que permitan realizar } \\
\text { consultas eficientes a la base de datos. }\end{array}$ & $N F$ & $T$ & $x$ \\
\hline REQNF-029 & $\begin{array}{l}\text { SCLOUDPY debe estar disponible las } 24 \text { horas del día para todo tipo } \\
\text { de usuario mediante clúster. }\end{array}$ & $N F$ & $T$ & $x$ \\
\hline REQNF-030 & $\begin{array}{l}\text { SCLOUDPY debe persistir los datos de los usuarios en una base de } \\
\text { datos. }\end{array}$ & $N F$ & $T$ & $x$ \\
\hline REQNF-031 & $\begin{array}{l}\text { SCLOUDPY debe ser instalado y ejecutado en los servidores de la } \\
\text { Institución. }\end{array}$ & $N F$ & $T$ & $x$ \\
\hline REQNF-032 & $\begin{array}{l}\text { SCLOUDPY debe funcionar en una máquina virtual en ambiente } \\
\text { Linux. }\end{array}$ & $N F$ & $B$ & $x$ \\
\hline REQNF-033 & $\begin{array}{l}\text { Se deben generar componentes de negocio y Web Services que } \\
\text { ofrezcan los servicios que tiene SCLOUD. }\end{array}$ & $N F$ & $T$ & \\
\hline
\end{tabular}


Posterior a la construcción de la tabla de requerimientos, se realizaron las siguientes actividades:

i) Crear una tabla de análisis comparativo para definir si un requerimiento es alcanzable, medible, y por qué es necesario;

ii) Aplicar un DAR (Decision Analisys and Resolution). Esta metodología DAR ayuda a analizar las posibles alternativas de decisión utilizando un proceso de evaluación formal que evalúa las alternativas respecto a un conjunto de criterios establecidos (Phifer, 2004);

iii) Elegir los requerimientos con los puntajes superiores;

iv) Diseñar un conjunto de componentes que representen la arquitectura informática que soporta las funcionalidades factibles que se van a incluir en el sistema SCLOUDPY;

v) Implementar los componentes arquitecturales que soportan las funcionalidades factibles de la extensión de SCLOUDPY;

vi) Validar en una empresa pequeña las nuevas funcionalidades implementadas para SCLOUDPY mediante pruebas de aceptación, y determinar si la aplicación cumple o no con las necesidades de la empresa.

\section{SELECCIÓN DE FUNCIONALIDADES}

Los requerimientos que se obtuvieron durante los análisis fueron plasmados en una tabla de análisis (ver tabla 3), que ayuda a definir si un requerimiento es alcanzable, medible, y por qué es necesario.

Algunos de los campos que incluye esta tabla son: a) Describir requerimiento: en la forma "SCLOUDPY debe..."; b) Justificación: por qué es necesario?; c) Factible: Por qué considera que es posible lograr este requerimiento?; y d) Medible: Cómo se medirá para verificar que el requerimiento se cumple?

Tabla 3. Análisis de Requerimientos del sistema SCLOUDPY: contenido parcial

\begin{tabular}{|c|c|c|c|c|}
\hline Código-Req & $\begin{array}{l}\text { Especificación Requerimiento: } \\
\text {-usar forma "el sistema debe . . } \\
\text {." } \\
\text { - no usar: "soportará", "no estará } \\
\text { limitado a..", "etc", "y/o", } \\
\text { "minimizar", "maximizar", } \\
\text { "rápido", "amigable", "fácil", } \\
\text { "suficiente", "adecuado" }\end{array}$ & $\begin{array}{l}\text { Justificación: } \\
\text { por qué es } \\
\text { necesario? }\end{array}$ & $\begin{array}{l}\text { Alcanzable: } \\
\text { Explique por qué es } \\
\text { factible construirlo }\end{array}$ & $\begin{array}{l}\text { Medible: } \\
\text { Cómo se medirá para } \\
\text { verificar que el } \\
\text { requerimiento se cumple? }\end{array}$ \\
\hline REQF-001 & $\begin{array}{l}\text { SCLOUD debe permitir la } \\
\text { gestión de inventarios para } \\
\text { PYMEs. }\end{array}$ & $\begin{array}{l}\text { Es necesario } \\
\text { porque las } \\
\text { PYMES deben } \\
\text { administrar de } \\
\text { forma correcta } \\
\text { los productos } \\
\text { que venden. }\end{array}$ & $\begin{array}{l}\text { Es posible lograr este } \\
\text { requerimiento porque } \\
\text { la persistencia de } \\
\text { información } \\
\text { relacionada con } \\
\text { inventarios de una } \\
\text { PYME facilita la } \\
\text { gestión de la misma. }\end{array}$ & $\begin{array}{l}\text { Se medirá utilizando los } \\
\text { servicios del sistema que } \\
\text { consultan y modifican } \\
\text { inventarios, verificando } \\
\text { que los datos sean } \\
\text { correctos. }\end{array}$ \\
\hline REQF-002 & $\begin{array}{l}\text { SCLOUD debe permitir a una } \\
\text { PYME hacer pedidos de } \\
\text { productos por lotes a un } \\
\text { proveedor. }\end{array}$ & $\begin{array}{l}\text { Es necesario } \\
\text { porque hoy en } \\
\text { día las PYMES } \\
\text { no realizan un } \\
\text { solo pedido } \\
\text { grande sino } \\
\text { que realizan } \\
\text { pedidos por } \\
\text { lotes. }\end{array}$ & $\begin{array}{l}\text { Es posible lograr este } \\
\text { requerimiento porque } \\
\text { se persistirá la } \\
\text { información } \\
\text { relacionada a } \\
\text { diferentes lotes de } \\
\text { productos que hagan } \\
\text { parte de un pedido. }\end{array}$ & $\begin{array}{l}\text { Se medirá verificando en } \\
\text { la base de datos la } \\
\text { información de entrega de } \\
\text { cada lote. }\end{array}$ \\
\hline
\end{tabular}

A partir de la tabla 3, se aplicó un DAR (Phifer, 2004) para seleccionar los requerimientos más relevantes respecto a un conjunto de criterios y subcriterios que aparecen en la tabla 4 . La tabla 5 muestra un ejemplo del DAR de selección con las calificaciones dadas por expertos. Los requerimientos que se definieron para hacer parte de las extensiones de SCLOUDPY son los que obtuvieron un puntaje mayor o igual a 4 en una escala Likert de 1 a 5 , y para este proyecto, 19 requerimientos de los 33 considerados cumplieron con esa validación y son los que aparecen en la tabla 2 marcados con $(X)$. 
Tabla 4. Resumen de criterios y subcriterios del sistema SCLOUDPY

\begin{tabular}{|c|c|c|c|c|c|}
\hline Criterio & Subcriterio & Descripción & Criterio & Subcriterio & Descripción \\
\hline \multirow[t]{2}{*}{ Correctitud } & Tiempo de vida & $\begin{array}{l}\text { Duración en } \\
\text { funcionamiento en } \\
\text { el sistema }\end{array}$ & \multirow[t]{2}{*}{ Eficiencia } & $\begin{array}{l}\text { Eficiencia de } \\
\text { Ejecución }\end{array}$ & $\begin{array}{l}\text { Nivel de } \\
\text { procesamiento } \\
\text { que necesita el } \\
\text { sistema para } \\
\text { cumplir con el } \\
\text { requerimiento. }\end{array}$ \\
\hline & $\begin{array}{l}\text { Completitud } \\
\text { (completitud del } \\
\text { sistema) }\end{array}$ & $\begin{array}{l}\text { Nivel de maduréz } \\
\text { que adquiere el } \\
\text { sistema con el } \\
\text { requerimiento. }\end{array}$ & & $\begin{array}{l}\text { Eficiencia de } \\
\text { Almacenamiento }\end{array}$ & $\begin{array}{l}\text { Minimiza el } \\
\text { espacio de } \\
\text { almacenamiento }\end{array}$ \\
\hline \multirow{2}{*}{ Usabilidad } & $\begin{array}{l}\text { Operabilidad (poca } \\
\text { exigencia de usuario) }\end{array}$ & $\begin{array}{l}\text { Qué tan complejo } \\
\text { puede ser el } \\
\text { manejo del } \\
\text { requerimiento para } \\
\text { un usuario. } \\
\end{array}$ & \multirow{2}{*}{ Mantenibilidad } & $\begin{array}{c}\text { Baja } \\
\text { Modularidad }\end{array}$ & $\begin{array}{c}\text { Nivel de } \\
\text { dependencia de } \\
\text { un requerimiento } \\
\text { para otros }\end{array}$ \\
\hline & $\begin{array}{c}\text { Poco entrenamiento de } \\
\text { Usuario }\end{array}$ & $\begin{array}{l}\text { Qué tan compleja } \\
\text { es la } \\
\text { familiarización del } \\
\text { usuario con el } \\
\text { requerimiento. }\end{array}$ & & $\begin{array}{c}\text { Bajo } \\
\text { Acoplamiento }\end{array}$ & $\begin{array}{l}\text { Nivel de } \\
\text { interacción con } \\
\text { otros } \\
\text { requerimientos. }\end{array}$ \\
\hline \multirow{2}{*}{ Funcionalidad } & \multirow{2}{*}{ Adecuación } & \multirow{2}{*}{$\begin{array}{c}\text { Nivel de } \\
\text { adaptación que } \\
\text { tiene el } \\
\text { requerimiento en } \\
\text { un cambio, sin } \\
\text { perder la calidad } \\
\text { de los servicios }\end{array}$} & \multirow{2}{*}{ Funcionalidad } & $\begin{array}{l}\text { cobertura de } \\
\text { roles (actual) }\end{array}$ & $\begin{array}{c}\text { Nivel de } \\
\text { afectación del } \\
\text { requerimiento a } \\
\text { los diferentes } \\
\text { roles del sistema. }\end{array}$ \\
\hline & & & & $\begin{array}{l}\text { Indispensable } \\
\text { para el Sistema }\end{array}$ & $\begin{array}{c}\text { Nivel de } \\
\text { importancia que } \\
\text { tiene el } \\
\text { requerimiento } \\
\text { para el sistema. }\end{array}$ \\
\hline
\end{tabular}

Tabla 5. DAR para seleccionar los requerimientos más relevantes para el sistema SCLOUDPY: contenido parcial

\begin{tabular}{|c|c|c|c|c|c|c|c|c|c|}
\hline \multicolumn{4}{|c|}{ Comparación de Alternativas } & \multirow{2}{*}{\multicolumn{3}{|c|}{$\begin{array}{l}\text { REQF-001 } \\
\text { SCLOUD debe permitir la } \\
\text { gestión de inventarios para } \\
\text { PYMEs. }\end{array}$}} & \multirow{2}{*}{\multicolumn{3}{|c|}{$\begin{array}{l}\text { REQF-002 } \\
\text { SCLOUD debe permitir a una } \\
\text { PYME hacer pedidos de } \\
\text { productos por lotes a un } \\
\text { proveedor. }\end{array}$}} \\
\hline & \multirow[b]{2}{*}{$\begin{array}{l}\text { 5: Totalmente de } \\
\text { acuerdo. } \\
\text { 1:Totalmente en } \\
\text { desacuerdo. }\end{array}$} & \multirow[b]{2}{*}{$\begin{array}{l}\text { Peso } \\
\text { Grupo }\end{array}$} & \multirow[b]{2}{*}{$\begin{array}{l}\text { Peso } \\
\text { criterio } \\
\text { (en el } \\
\text { grupo) }\end{array}$} & & & & & & \\
\hline & & & & \multirow[t]{2}{*}{ asesor1 } & \multirow[t]{2}{*}{ asesor2 } & asesor3 & \multirow[t]{2}{*}{ asesor1 } & \multirow[t]{2}{*}{ asesor2 } & asesor3 \\
\hline Correctitud & & $20 \%$ & & & & & & & \\
\hline & Trazabilidad & & $60 \%$ & 4 & 5 & 4 & 5 & 5 & 4 \\
\hline & Completitud & & $40 \%$ & 3 & 5 & 4 & 4 & 5 & 5 \\
\hline & Subtotal & & $100 \%$ & 3.60 & 5.00 & 4.00 & 4.60 & 5.00 & 4.40 \\
\hline \multirow[t]{4}{*}{ Usabilidad } & & $20 \%$ & & & & & & & \\
\hline & $\begin{array}{l}\text { Operabilidad (poca } \\
\text { exigencia de } \\
\text { usuario) }\end{array}$ & & $65 \%$ & 3 & 3 & 3 & 5 & 5 & 4 \\
\hline & $\begin{array}{l}\text { Poco entrenamiento } \\
\text { de Usuario }\end{array}$ & & $35 \%$ & 3 & 3 & 3 & 4 & 5 & 4 \\
\hline & Subtotal & & $100 \%$ & 3.00 & 3.00 & 3.00 & 4.65 & 5.00 & 4.00 \\
\hline \multirow[t]{4}{*}{ Eficiencia } & & $20 \%$ & & & & & & & \\
\hline & $\begin{array}{l}\text { Eficiencia de } \\
\text { Ejecución }\end{array}$ & & $70 \%$ & 3 & 3 & 2 & 5 & 2 & 5 \\
\hline & $\begin{array}{l}\text { Eficiencia de } \\
\text { Almacenamiento }\end{array}$ & & $30 \%$ & 2 & 1 & 2 & 5 & 4 & 5 \\
\hline & Subtotal & & $100 \%$ & 2.70 & 2.40 & 2.00 & 5.00 & 2.60 & 5.00 \\
\hline \multirow[t]{4}{*}{ Mantenibilidad } & & $10 \%$ & & & & & & & \\
\hline & Alta Modularidad & & $65 \%$ & 3 & 4 & 4 & 3 & 3 & 3 \\
\hline & Bajo Acoplamiento & & $35 \%$ & 2 & 4 & 4 & 4 & 3 & 3 \\
\hline & Subtotal & & $100 \%$ & 2.65 & 4.00 & 4.00 & 3.35 & 3.00 & 3.00 \\
\hline
\end{tabular}


Tabla 5: (continuación)

\begin{tabular}{|c|c|c|c|c|c|c|c|c|c|}
\hline Funcionalidad & & $30 \%$ & & & & & & & \\
\hline & Adecuación & & $30 \%$ & 2 & 4 & 2 & 5 & 3 & 5 \\
\hline & $\begin{array}{l}\text { cobertura de roles } \\
\text { (actual) }\end{array}$ & & $30 \%$ & 4 & 4 & 3 & 4 & 3 & 5 \\
\hline & $\begin{array}{l}\text { Indispensable para } \\
\text { el Sistema }\end{array}$ & & $40 \%$ & 2 & 1 & 3 & 4 & 3 & 5 \\
\hline & Subtotal & & $100 \%$ & 2.60 & 2.80 & 2.70 & 4.30 & 3.00 & 5.00 \\
\hline & \multicolumn{3}{|c|}{$\begin{array}{r}\text { Calificación ponderada de cada } \\
\text { asesor: }\end{array}$} & 2.91 & 3.32 & 301 & 448 & 3.72 & 448 \\
\hline & \multicolumn{3}{|c|}{ TOTALES $100 \%$} & & 3.08 & & & 4.23 & \\
\hline
\end{tabular}

Posteriormente para diseñar e implementar las extensiones propuestas para SCLOUDPY como sistema informático, se siguió la metodología de Programación Extrema (XP) aprovechando que es una metodología ágil. XP pretende llevar buenas prácticas de la Ingeniería de software pero al extremo, según Kent Beck (Beck, 2000; XP, 2015), tal como se ilustra en la figura 1. Las extensiones se implementaron bajo una Arquitectura informática que soporta los requerimientos que cumplen el criterio de selección. Con este fin se tomaron las funcionalidades definitivas de la extensión del prototipo de SCLOUDPY y se implementó una lógica de negocio apoyándose en la suite de gestión de procesos de negocio BonitaSoft (BOS) (Bonita, 2015) la cual opera sobre un servidor de aplicaciones JBoss (JBoss, 2015).

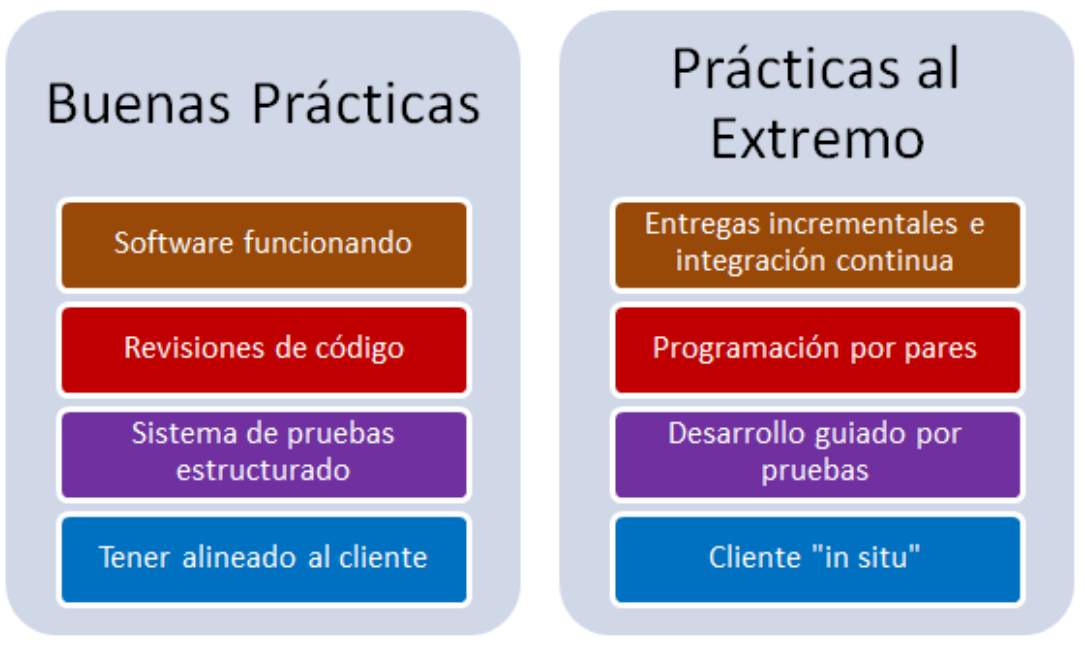

Fig. 1: Metodología de programación extrema (XP,2014)

\section{ARQUITECTURA DE MULTI-TENENCIA DEL SISTEMA SCLOUDPY}

En la implantación informática del modelo SCLOUDPY en su versión inicial era necesario realizar una serie de configuraciones y despliegues adicionales por cada pyme que quisiera utilizar el sistema que se realizaban dentro de la misma máquina en la que estaba la instancia del servidor con la lógica de negocio. Esta arquitectura con múltiples pymes era ineficiente, pues los recursos de la máquina servidor que lo alojaría se gastarían de una forma más rápida de acuerdo a la cantidad de pymes que debían estar en funcionamiento; es decir que para tener una mayor cantidad de Pymes era necesario tener un servidor JBoss con una IP dedicada por cada pyme (Corzo, 2014). Por esta razón se planteó una nueva arquitectura que evitara el gasto de recursos por cada pyme y que solo requiriese el despliegue de dos instancias del servidor de aplicaciones JBoss, uno para la lógica de negocio y otro para la suite BOS para la presentación e interacción con los usuarios de múltiples pymes. Esta nueva arquitectura pretende ser realmente de tenencia múltiple (multi-tenencia) soportando múltiples pymes a la vez a través de una sola aplicación y asegurando a la vez la separación de los datos de cada pyme.

Con la arquitectura planteada fue necesario realizar cambios en múltiples componentes del sistema; en primer lugar se debió realizar una refactorización de la lógica de negocio, con el fin de que cada usuario indique la pyme a la cual pertenece en el momento de la autenticación y a partir de ahí todos los servicios que solicite se ejecuten únicamente con los datos de esa pyme. A partir de estos cambios de la lógica de negocio también por consecuencia se debieron realizar cambios en la base de datos para asegurar la separación de la información de cada pyme. 
También se realizó una refactorización sobre todos los procesos de negocio que se habían desarrollado en SCLOUDPY en su versión inicial, debido a que inicialmente estos procesos al igual que el servidor JBoss de Bonita estaban programados para ejecutarse para una sola pyme en particular, siendo necesario otra instancia de servidor JBoss para una segunda pyme. La actual arquitectura mejora la anterior, debido a que ya no existe la necesidad de desplegar varios servidores JBoss de BOS, uno para cada pyme, evitando un gasto de recursos muy grande. La figura 2 ilustra la arquitectura de software para el conjunto de pymes (tenants) utilizando la suite BPMS BonitaSoft Open Solution (Bonita, 2015) en donde se requieren únicamente 2 servidores JBoss: uno para ejecutar el motor de procesos de negocio (Bonita Execution Engine) y otro para ejecutar la lógica de negocio que accede a la base de datos y que es compartida por el conjunto de pymes. El motor de procesos de negocio ejecuta los flujos de información derivados de los procesos BPMN (BPMN, 2015) para un Cliente, para una Pyme y para el MRP.

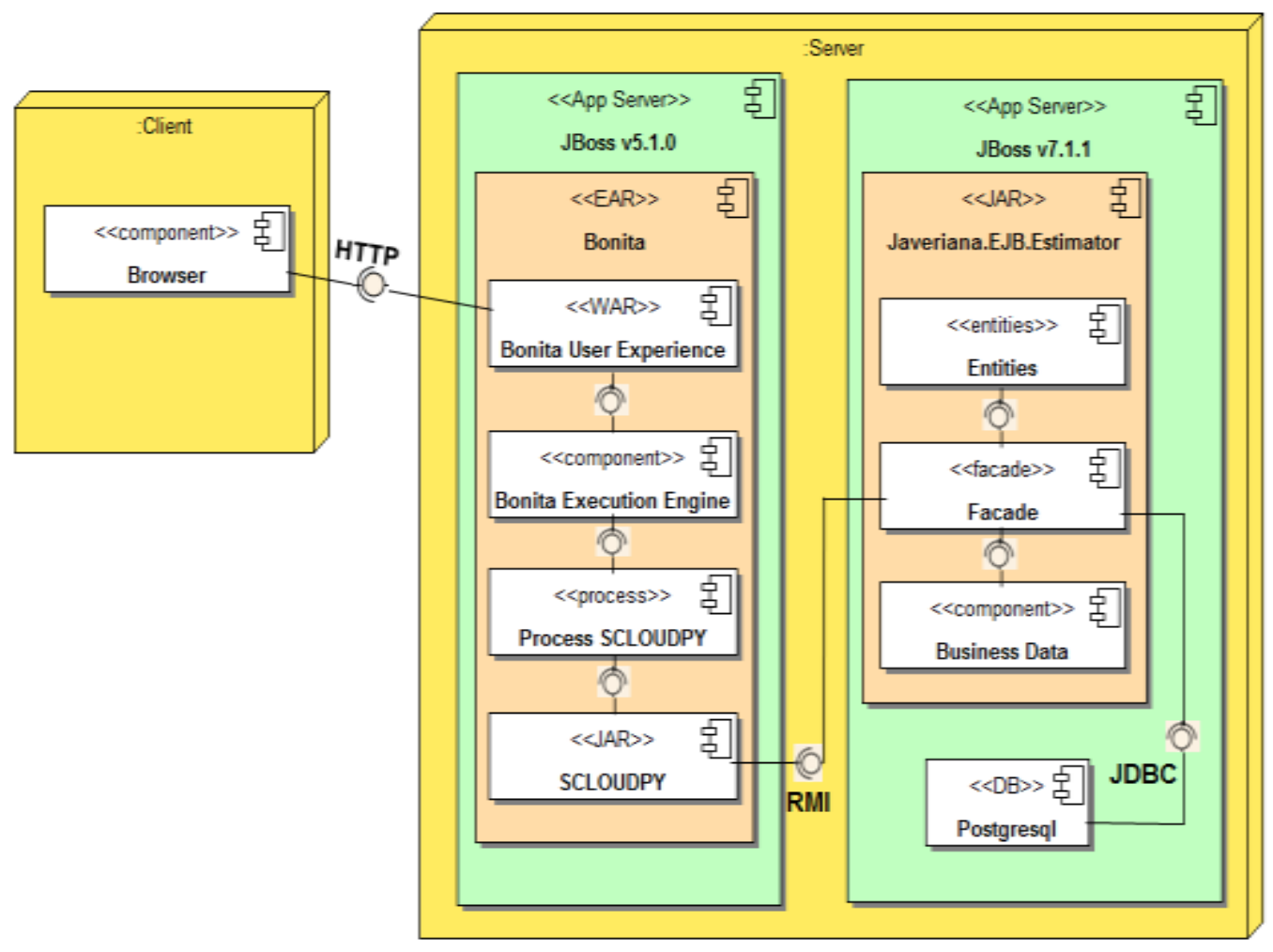

Fig. 2: Arquitectura multi-tenencia del sistema SCLOUDPY ( Corzo, 2014)

Todas la Pymes comparten el motor de procesos de negocio, Bonita Execution Engine y los usuarios invocan SCLOUDPY desde un navegador de Internet. Cada pyme ignora los detalles técnicos de la operación del sistema en modo "Computación en la nube" con múltiple tenencia. En este modo también es transparente para una pyme que sus datos convivan en la misma base de datos en donde residen los datos de las demás pymes. Esta nueva arquitectura pretende ser realmente multi-tenencia soportando múltiples pymes a la vez a través de una sola aplicación pero asegurando la separación de los datos de cada pyme.

A cada uno de los procesos de negocio se le modificaron los "conectores" que hacen los llamados a la lógica de negocio implantada mediante componentes Java EJB (Java, 2015), en donde se implementó una nueva forma de identificación del usuario, para poder determinar de qué pyme es la información que se está manejando. En la figura 3 se muestra el nuevo modelo de datos (entidades de negocio) de la actual versión del sistema SCLOUDPY.

\section{Configuración de la máquina virtual para SCLOUDPY}

Se decidió mejorar la máquina virtual alojada en el servidor. Esto debido a que la máquina virtual en la versión inicial de SCLOUDPY tenía Ubuntu (Ubuntu, 2015) edición para escritorio como sistema operativo y esto afectaba el rendimiento debido a que usa un entorno gráfico que consume más recursos de máquina y adicionalmente software que no es necesario. Se escogió CentOS (T.C.Project,2015) versión 6.6 como nuevo sistema operativo para la máquina virtual debido a que es mucho más ligero que el anterior, además de ser un sistema operativo especializado para ser servidor. Este es uno de los servidores más usados en el mundo Linux y adicionalmente es software libre. 
La nueva máquina virtual fue instalada con las siguientes características que podrían ser ampliadas cuando el sistema SCLOUDPY opere para un número grande de pymes: a) Procesador: 4 Núcleos; b) Memoria RAM:6 GB; y c) Disco Duro: 60 GB. En esta máquina virtual están desplegados los servidores de aplicación que componen el sistema SCLOUDPY para realizar sus diferentes tipos de operaciones. La máquina está disponible con una dirección IP que solo es accesible dentro de la red de área local (LAN) de la institución para realizar las pruebas pertinentes sobre el sistema; en el futuro el servidor con esta dirección IP será puesto al acceso público a través de internet para ser utilizado por las pequeñas y medianas empresas que estén interesadas.

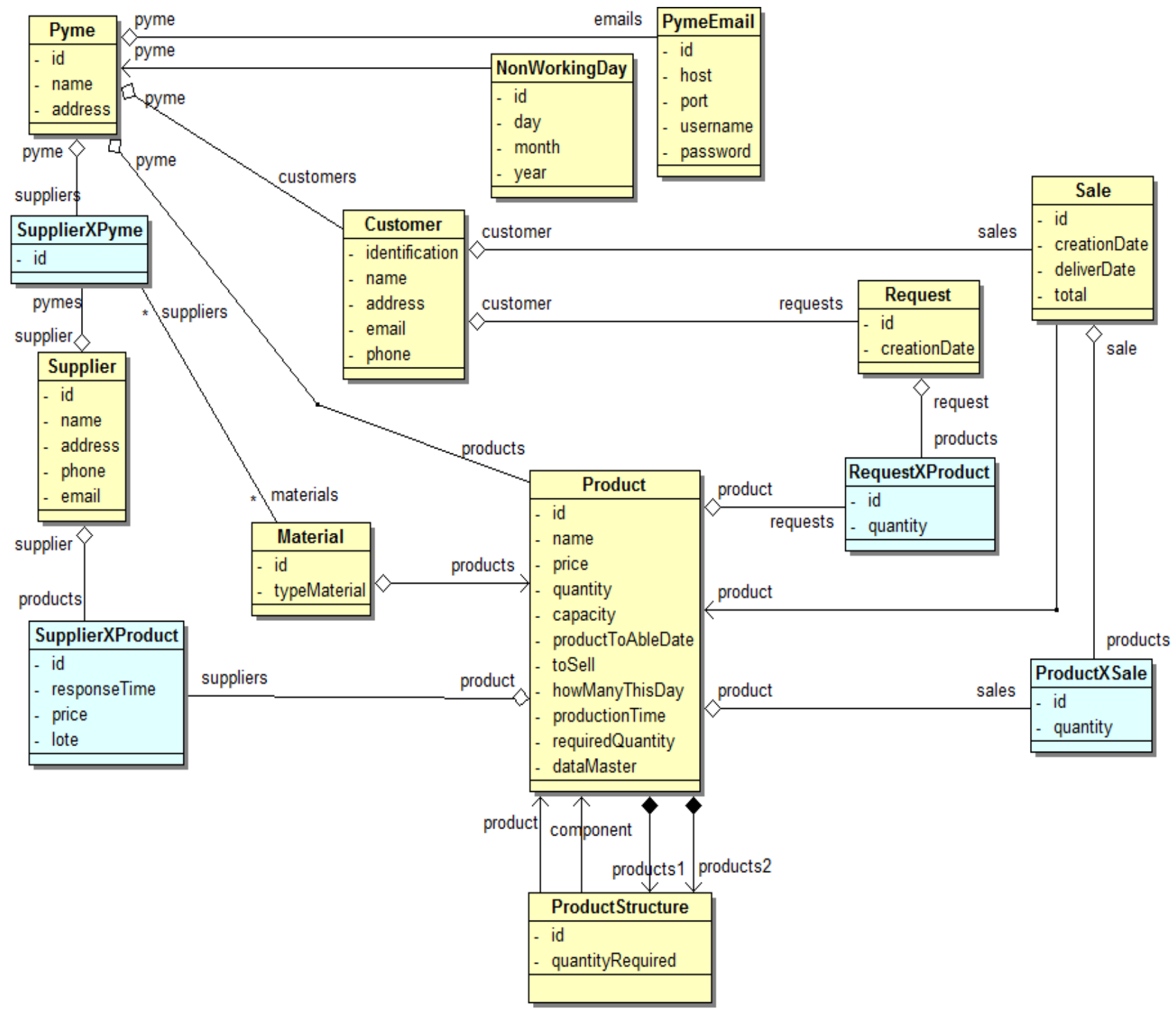

Fig. 3: Modelo de entidades de negocio de SCLOUDPY (Corzo, 2014)

\section{VALIDACION}

\section{Prueba de carga de usuarios}

A través de la herramienta JMeter (Apache Software Foundation, 2015) se diseñó una prueba simulando diferentes usuarios de diferentes empresas pymes. Consistía en realizar un inicio de sesión en el sistema SCLOUDPY, con el fin de medir el rendimiento del sistema con respecto a la capacidad de manejo de múltiples usuarios. Específicamente la prueba consistió en enviar solicitudes de inicio de sesión al servidor en el menor tiempo posible, cada petición con un usuario y contraseña diferente, con esto se midió el tiempo de respuesta del servidor arrojando los datos que se observan en la figura 4. El tiempo para 1000 usuarios fue menor a medio segundo lo que indica un buen desempeño en este sentido.

\section{Prueba de carga de flujo de pedido}

Para este caso se definió hacer la prueba del proceso completo de realizar la solicitud de un pedido; se inició el proceso con una (1) pyme haciendo una solicitud y se llevó hasta 40 pymes. El resultado promedio 
de tiempos por solicitud para 1,5,10,20,30,40 pymes, es el que se muestra en el siguiente figura 5 ( Corzo,2014).

El tiempo de ejecución del proceso de la solicitud de una compra de un cliente puede ser bastante largo si el sistema esta sobrecargado; al atender 5 solicitudes de pedido diferentes de usuarios de varias pymes, el tiempo de respuesta es excesivo debido a que supera los 40 segundos. Se observa en la figura 5 que para atender 40 solicitudes correctamente el tiempo se eleva a 676 segundos, y la línea de tendencia indica un aumento potencial.

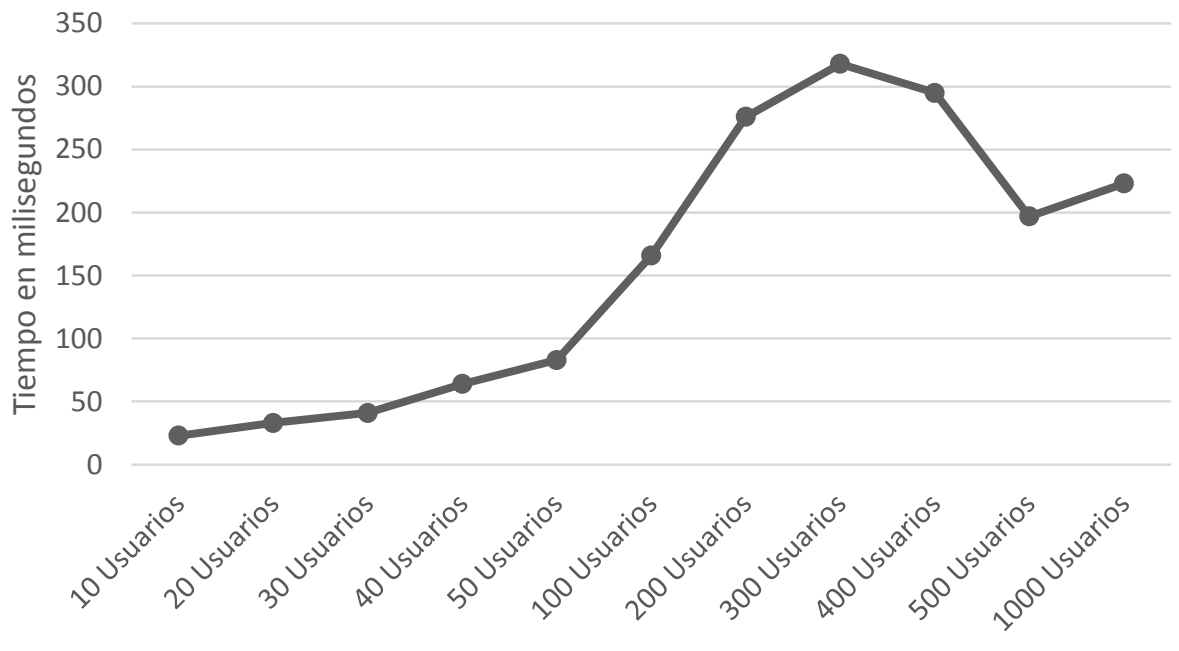

Numero de usuarios inicio de sesión

Fig. 4: Promedio de ms por número de usuarios (Corzo, 2014)

Es posible que estos tiempos tan altos para atender las solicitudes se deban a la velocidad del procesador de la máquina virtual; aumentar esta velocidad puede disminuir el tiempo de respuesta en este proceso de negocio en varios segundos, por lo que sería interesante volver a identificar los tiempos con un procesador mucho más veloz y así determinar cuántas peticiones se pueden atender al mismo tiempo, con un tiempo de respuesta aceptable.

También es importante resaltar que el algoritmo que maneja lo lógica de negocio del MRP (Planificación de los requerimientos de material) tiene una complejidad bastante alta, debido al manejo de cada uno de los productos y su composición que afectan directamente el rendimiento del sistema. Es decir dependiendo de la composición de materiales de un producto el sistema puede responder de una mejor o peor manera a las solicitudes, por lo que sería importante pensar en la posibilidad de hacerle reformas a este algoritmo.

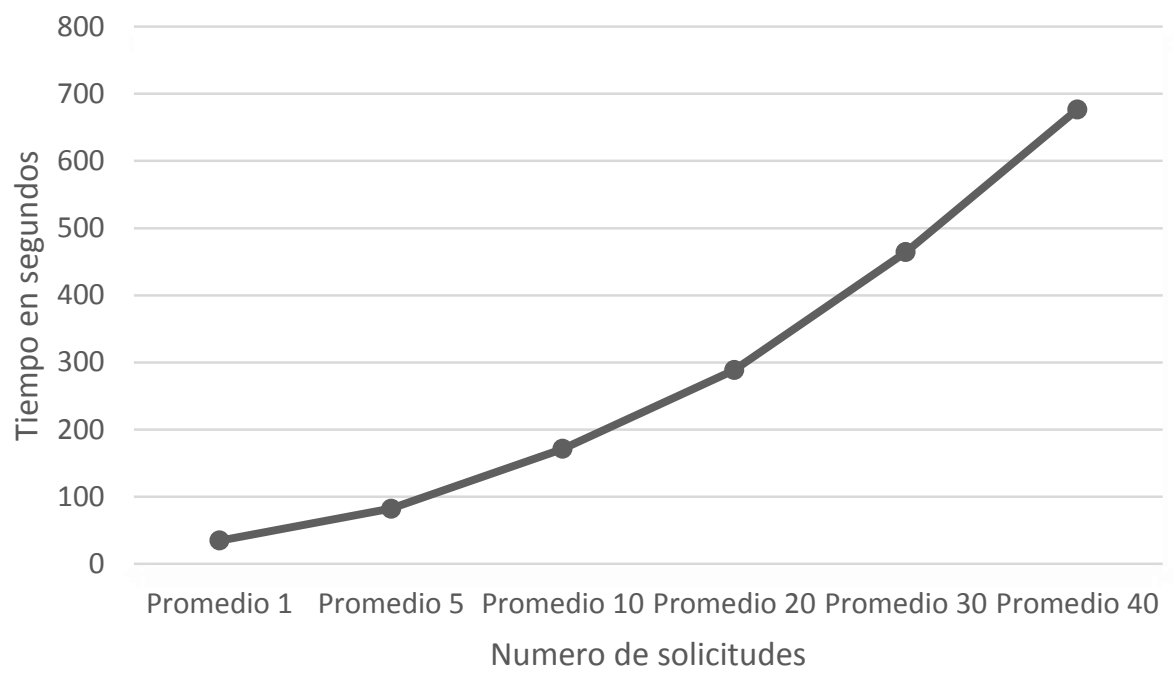

Fig. 5. Gráfico de tiempo en segundos por número de usuarios (Corzo, 2014) 


\section{CONCLUSIONES}

Un sistema como SCLOUDPY para la gestión del flujo de pedidos de pymes manufactureras constituido con software libre y con características multi-tenencia, permite ofrecer soluciones a las Pymes que generalmente cuentan con recursos limitados. Además son pocos los sistemas de Procesamiento de pedidos que se implantan siguiendo esta arquitectura debido a la incertidumbre que las empresas tienen respecto a esta tecnología en los aspectos de seguridad; SCLOUDPY resuelve la seguridad de la aplicación apoyándose en las facilidades de autenticación y autorización que ofrece la suite de gestión de procesos de negocio BonitaSoft (BOS) y utilizando la identificación de la pyme suministrada en la autenticación como filtro en todas las consultas a la base de datos con el fin de que cada pyme no pueda acceder a datos de otra pyme. Las posibles funcionalidades de un sistema como SCLOUDPY son múltiples, sin embargo en una primera etapa y con recursos limitados, es necesario definir cuáles de ellas son realmente vitales para el uso de las empresas.

El sistema tiene un tiempo de respuesta apropiado. Sin embargo, las pruebas de carga con solicitudes de flujo de pedido (todo el proceso), indicaron un crecimiento exponencial en el tiempo de respuesta, que implica un tiempo de servicio alto para los usuarios aunque sigue siendo mucho mejor que el desempeño manual frecuentemente implementado por las pymes. Se podría replantear el algoritmo que realiza la estimación de los materiales (MRP) para que sea más eficiente pues con el sistema actual sus tiempos de respuesta no son los mejores. En el estado actual el sistema SCLOUDPY cuenta con una arquitectura mejorada donde ya no existe la necesidad de desplegar varios servidores de aplicaciones, uno para cada pyme, sino únicamente 2 servidores en total, evitando un gasto de recursos muy grande; en el primer servidor se ejecuta el motor de procesos de negocio (Bonita Execution Engine) y en el segundo se ejecuta la lógica de negocio que accede a la base de datos y que es compartida por el conjunto de pymes.

En el estado actual SCLOUDPY opera como máquina virtual sobre el sistema operativo Linux CentOS versión 6.6 que es más ligero y robusto que el sistema operativo Ubuntu edición escritorio que se utilizó en la versión anterior de SCLOUDPY. SCLOUDPY como sistema que opera bajo el modelo de operación en la Nube goza de las ventajas que trae este tipo de tecnología, permitiendo un uso óptimo de recursos mediante la eventual reconfiguración dinámica del sistema de acuerdo a las necesidades de la empresa o empresas que utilicen el sistema.

\section{REFERENCIAS}

AbilityOMS, Order Management System Ability OMS,

http://www.abilitycommerce.com/ProductsSolutions/OrderManagement/AbilityOMS.aspx. Acceso 27 de abril (2012)

Adempiere, Adempiere, http://www.adempiere.com/ADempiere_ERP. Acceso 22 de junio (2015)

Aguilar-Saven, R., Business process modeling: review and framework. International Journal of Production Economics, Vol. 90 No. 2, 129-149 (2003)

Antoniadis, I., Tsiakiris, T., Tsopogloy S., Business Intelligence during times of crisis: Adoption and usage of ERP systems by SMEs. International Conference on Strategic Innovative Marketing, IC-SIM 2014, September, Madrid, Spain (2014)

Amer, Y., Luang, L. y Lee, S., Case study: optimizing order fulfillment in a global retail supply Chain, International Journal of Production Economics, 35, 14-17(2009)

Apache Software Foundation, Apache JMeter, https://jmeter.apache.org/index.html. Acceso 22 de junio (2015)

Asociación de Contabilidad y administración de Empresas, Sistemas de Información Integrados USA (ERP), https://www5.uva.es/guia_docente/uploads/2012/430/52298/1/Documento8.pdf. Acceso 22 de junio (2015)

Beck, K. y Fowler, M., Planning Extreme Programming, Addison-Wesley Professional -XP Series, (2000)

Bonita, BonitaSoft Documentacion, http://www.bonitasoft.com/resources/documentation. Acceso 22 de junio (2015)

Boyer, K.K. and Hult, G.T.M., Extending the supply chain: integrating operations and marketing in the online grocery industry, Journal of Operations Management, Vol. 23 No. 6, 642-61 (2005) 
BPMN, The tools to manage your business, http://ordermanagementsystems.com.au/. Acceso 22 de Junio (2015)

Bruegge, B., Dutoit: Ingeniería de Software Orientado a Objetos. Prentice Hall (2002)

Aslan, B., Stevenson, M., Hendry, L., Enterprise Resource Planning systems: An assessment of applicability to Make-To-Order companies, Computers in Industry, 63, 692-705 (2012)

Cabarcas, A., Puello, P., Martelo, R., Sistema de Información Soportado en Recuperación XML para Pequeñas y Medianas Empresas (PYME) de Cartagena de Indias, Colombia. Información tecnológica., 26(2), 135-144, (2015)

Carrillo, M. y Franky. M., Modelo SCLOUDPY para la gestión de pedidos en la nube, Información Tecnológica, 25(4), 35-42 (2014)

Cegielski, C. G., Jones-Farmer, L. A. Wu, Y., Hazen, B. T., Adoption of cloud computing technologies in supply chains, The International Journal of Logistics Management, Vol. 23, 184 - 211 (2012)

Corzo, C.,SCLOUD-MT: Actualización y transformación del sistema SCLOUD hacia una versión con soporte para varias empresas (multitenant) sin restricciones. Trabajo de grado de Ingeniería de Sistemas, Facultad de Ingeniería, Pontificia Universidad Javeriana, Bogotá (2014)

Chike, O. y M. Xiong, A decisión support system framework to process customer order enquiries in SMEs. International Journal of Advanced Manufacturing Technology, 42, 398-407 (2009)

Christopher, M., Lowson, R. Peck, H., Creating agile supply chains in the fashion industry, International Journal of Retail and Distribution Management, Vol. 32 No. 8, 367-76 (2004)

Dynamics, Microsoft Dinamics. http://www.microsoft.com/en-us/dynamics/default.aspx. Acceso 22 de junio (2015)

Elragala, A., Haddarab,M., The Future of ERP Systems: look backward before moving, CENTERIS 2012 Conference on ENTERprise Information Systems ( 2012)

EPICOR, EPICOR Business Inspired,

http://discover.epicor.com/lac/erp/sp/ggl?epi_sourcecode=ERPA_CO_SEM_GOGL_FY13_ERP\&utm_sourc e=google\&utm_medium =cpc\&utm_campaign $=$ CO_-

_EPIC_ERP_ES\&utm_term=erp\&utm_content=covepi25p5787. Acceso 22 de junio (2015)

ERP5, Open source MRP, http://www.erp5.com/. Acceso 22 de junio (2015)

Garcia, D., You, F., Supply chain design and optimization: Challenges and opportunities. Computers and Chemical Engineering, 81, 153-170 (2015)

Hsu, $\mathrm{P}$, Integrating ERP and e-business: Resource complementarity in business value creation. Decision Support Systems 56, 334-347(2013)

IPO Board, Doing Business in Russia,

https://www.google.com.co/url?sa=t\&rct=j\&q=\&esrc=s\&source=web\&cd=2\&cad=rja\&ved=0CEIQFjAB\&url=ht tp\%3A\%2F\%2Fwww.ipoboard.ru\%2Ffiles\%2Fcompanies\%2Fpublic\%2Febf87f30a466698a399dffc273e7719 3\&ei=Vyd2UZ_qNYjl0gHdsoDwAw\&usg=AFQjCNEFOVm1RZf7ls0_xi_z2iZyAZBWqg\&bvm=bv.45512109,d. $\mathrm{dmQ}$. Acceso 15 mayo (2012)

Jacobs, R., Weston, T., Enterprise resource planning (ERP)—A brief history. Journal of Operations Management 25, 357-363 (2007)

Java, Java Platform Enterprise Edition (Java EE),

http://www.oracle.com/technetwork/java/javaee/overview/index.html. Acceso 22 de junio (2015)

JDA, MANUGISTIC: software for the supply chain, http://www.jda.com/solutions/solutions-index/. Acceso 4 de mayo (2012) 
JBoss, What does jBPM do? http://www.jboss.org/jbpm. Acceso 22 de junio (2015)

Kshetri N., Cloud Computing in Developing Economies, Research Feature Published by the IEEE Computer Society (2010)

Lin, H., Zhu, X., Wang, P., Dong, X., Li, W., A service oriented supply chain collaboration system for SMEs, Industrial Electronics and Applications (ICIEA), 2011 6th IEEE Conference on (2011)

Lindner, M., Galán, F., Chapman C., Clayman, S., Henriksson, D., Elmroth, E., The Cloud Supply Chain: A Framework for Information, Monitoring, Accounting and Billing, CloudComp 2010, 2nd International ICST Conference on Cloud Computing, 2010 Barcelona, España (2010)

Logility, Solutions: Achieve a competitive advantage with collaborative supply chain solutions (2007), http://www.logility.com/solutions. Acceso 22 de junio (2015)

Logismarket, Advanced Planning, http://www.logismarket.com.mx. Acceso 12 de agosto (2014)

Neogia, Open source ERP, http://www.neogia.org/wiki/index.php/Portada. Acceso 20 de febrero (2012)

Open bravo,Commerce Suite, http://www.openbravo.com/ . Acceso 29 de marzo (2012)

OasisCom, OasisCom, http://www.oasis.com.co/ Acceso 9 de junio (2013)

OpenERP, Open ERP, http://v6.openerp.com/taxonomy/term/130. Acceso 18 de abril (2012)

OpenERP, Site original da comunidade da Localização Brasileira do OpenERP, http://www.openerpbrasil.org/. Acceso 22 de junio (2015)

Phifer, B., DAR Basics: Applying Decision Analysis and Resolution in the Real World, EDS (2004)

Productivos Itda, Factory el software industrial, http://catalogodesoftware.com/anunciante.aspx?aid=263. Acceso 22 de junio (2015)

Ruivo, P., Tiago, O., Neto, M. Using resource-based view theory to assess the value of ERP commercialpackages in SMEs. Computers in Industry 73,105-116 (2015)

Ruivo, P, Johansson, B.,Oliveira, T.,Neto, M. Commercial ERP systems and user productivity. CENTERIS 2013 - Conference on ENTERprise Information Systems (2013)

SAP, Fine-tune supply chain management, http://www.sap.com. Acceso 22 de junio (2015)

Siigo, SIIGO Pyme, http://www.siigo.com/pyme . Acceso 22 de junio (2015)

Su. Y., Yang Ch., Why are enterprise resource planning systems indispensable to supply chain management? . European Journal of Operational Research 203, 81-94 (2010)

T. C. Project, CentOS Project, http://www.centos.org. Acceso 22 de junio (2015)

Ubuntu, Ubuntu PC operating System, http://www.ubuntu.com/. Acceso 22 de junio (2015)

XP, Introducción agil a eXtreme Programming, http://www.slideshare.net/chileagil/introduccin-gil-a-extremeprogramming-webprendedor08-350127. Acceso 22 de junio (2015)

Yoshihara, H., Japanese companies and ERP: a study on information technology and manegerial style, http://iceb.nccu.edu.tw/proceedings/APDSI/2004/pdf/073.pdf. Acceso 22 de junio (2015)

You, J., Order Collaborative Management System for Small and Medium-sized Customization Enterprises. IEEE Xplore, 1-3 (2011)

Zare, Y., Enterprise Resource Planning: risk and benefit analysis. Business Strategy Series, 11, 308-324 (2010) 
